\title{
CORRELATOS ELECTROENCEFALOGRÁFICOS DURANTE LA DRAMATIZACIÓN DE UN CUENTO INFANTIL DE NATURALEZA REPETITIVA
}

\author{
Marzia Fiorini. Universidad de Cassino \\ Lucio Inguscio. Universidad de Roma "La Sapienza" \\ José Miguel García Ramírez. Universidad de Granada
}

Fecha de recepción: 20 de julio de 2014

Fecha de revisión: 30 de julio de 2014

Fecha de aceptación: 3 de agosto de 2014

\section{Resumen}

Los correlatos cerebrales del pensamiento creativo han sido investigados en muchos estudios que se han focalizado sobre el concepto de creatividad entendida como pensamiento divergente, con respecto del pensamiento convergente. Del análisis de estos factores se evidencia que la productividad divergente es una de las habilidades mentales que el sujeto utiliza y que lo distingue de los demás y se identifica el proceso creativo con la dinámica típica de la resolución de problemas. En este estudio se investiga la presencia de distintos patrones neurofisiológicos que subyacen en la capacidad, creativa o no, de solucionar un problema. Se pretende averiguar si la activación cortical de las áreas posteriores también participa en la resolución de tareas de naturaleza repetitiva y si tal activación, en términos de ritmo alpha, es significativamente más amplia en el momento en que los sujetos aprenden una estrategia optima de solución. Los participantes intentan realizar una tarea de naturaleza repetitiva; se analiza el momento inicial en el que los participantes tratan de realizarla de modo repetitivo, pensamiento convergente; posteriormente se vuelve a analizar después de que los sujetos hayan aprendido una estrategia optima, pensamiento divergente.

Palabras clave: Pensamiento divergente, potenciales relacionados a eventos, sincronización/desincronización relacionada a eventos, funciones ejecutivas

La creatividad es clave en la evolución de la civilización. Todos los descubrimientos e innovaciones científicas dependen de la habilidad de cambiar modelos de pensamiento, romper con el presente y construir algo nuevo. Esta extraordinaria capacidad de la mente humana es estudiada ampliamente tanto en las ciencias del comportamiento como en las neurociencias. Por lo que se han realizado notables progresos en las investigaciones realizadas en los últimos 50 años. Sin embargo, el objeto mismo de estudio "el pensamiento creativo" es difícil de investigar por la metodología clásica. El motivo principal de esta dificultad está en pedir al sujeto experimental ser creativo, instante del insight, en condiciones experimentales controladas. Esto no significa que en los últimos años no se hayan recogido datos de estudios de casos, entrevistas, formularios de personalidad y se hayan localizado los rasgos y las costumbres tanto de las personas creativas como de los modelos comunes que acompañan los procesos creativos. Sin embargo, las investigaciones sobre los procesos creativos realizados en los laboratorios de psicología no han producido la misma cantidad de datos.; aunque las investigaciones más recientes han 
mostrado más interés respecto a los mecanismos cerebrales implicados en el pensamiento creativo. (Dietrich, 2004; Bowden, Jung-Beeman, Fleck \& Kounios, 2005; Fiorini, \& Garcia-Ramirez, 2013)

Las investigaciones neurocientíficas sobre creatividad ven evolucionando y tomando importancia a partir de los primeros conceptos de la hipótesis del proceso primario de cognición (Kris,1952), la hipótesis de la atención de-focalizada (Mendelsohn,1976) y la hipótesis de las jerarquías asociativas (Mednick, 1962). Las personas creativas son capaces de cambiar rápidamente el pensamiento de la modalidad secundaria (pensamiento abstracto, lógico y analítico) a la modalidad primaria (sueño o imaginación) o bien son capaces de retroceder hacia los procesos de cognición primaria necesarios para producir narraciones o ideas originales. Las personas creativas también se caracterizan por la capacidad de proveer asociaciones a un dato estímulo lo más ampliamente posible "flat", con respecto a una modalidad más rígida "steep" que solo permite asociaciones comunes y en menor número de frecuencia respecto a un dato estímulo. (Fiorini, 2012)

Los procesos cognitivos primarios, atención de-focalizada y jerarquías asociativas de tipo "flat" ocurren todas las veces que el individuo se encuentra en un estado de baja activación "arousal cortical"; según esta hipótesis los individuos creativos muestran bajos niveles de arousal cortical respecto a individuos pocos creativos. (Martindale, 1999)

Los correlatos cerebrales del pensamiento creativo han sido usados en estudios que focalizados en el concepto de creatividad entendida como pensamiento divergente, con respecto del pensamiento convergente. Con el pensamiento divergente un problema se puede solucionar en modos diferentes, mientras con el convergente, se pretende descubrir la mejor o única solución a un problema. (Guilford, 1950,1967; Fiorini, 2012)

En una investigación realizada por Molle y colaboradores (1999) se pidió a un grupo de participantes que enumeraran de modo inusual el uso de objetos comunes y que imaginaran en diferentes situaciones hipotéticas en las que podrían ser utilizados (situación de pensamiento divergente); en cambio, en la condición de pensamiento convergente, se les indicó a los sujetos que completaran una secuencia de letras con una palabra dotada de significado. Molle y colaboradores (1999) elaboraron un cuadro electroencefalográfico de gran complejidad de los sujetos que se encontraban en la condición de pensamiento divergente con respecto de la del pensamiento convergente; la complejidad EEG en la primera condición mostró actividad desincronizada de algunas áreas cerebrales; del mismo modo Jausovec (2000), confirmó la hipótesis de una preponderancia del ritmo alpha durante el desarrollo de tareas creativas, predominante en las áreas centro-parietales. Sucesivamente se fue confirmado la hipótesis de la activación alpha, adjunta a tareas correlacionada con la experiencia del insight: el análisis del cuadro EEG muestra, sobre todo, altos valores de amplitud del ritmo alpha en situaciones de insight en el hemisferio derecho y en las regiones posteriores de la corteza (Bowden \& Jung-Beeman, 1998, 2003). El papel del hemisferio derecho también fue definido en los estudios que se ocuparon del pensamiento divergente durante el proceso de imaginación tanto narrativa como gráfica. (Martindale et at., 1978, 1984)

Este estudio se focaliza sobre la posibilidad de registrar un cuadro EEG con un sistema a alta densidad de registros, el sistema Geodesic, Net AMP 300 GES de 128 canales de grabación, pudiéndose valorar el curso de otros índices electroencefalográficos, asimetría cortical, sincronización y desincronización 
relacionada a eventos (ERS y ERD), ritmos más rápidos de tipo gamma y potenciales relacionados a eventos (ERPs). Contrariamente a los estudios anteriores, se pretende averiguar si la activación cortical de las áreas posteriores también participa en la resolución de tareas de naturaleza repetitiva y si tal activación, en términos de ritmo alpha, es significativamente más amplia en el momento en que los sujetos aprendan la estrategia de solución para realizar la tarea con el menor número posible de movimientos. A los participantes se les propone dramatizar un cuento de naturaleza repetitiva. Posteriormente se analizan dos momentos, uno inicial en el que los participantes tratan de realizar la tarea a modo "ensayo y error", de modo repetitivo (pensamiento convergente) y otro en el que los sujetos han aprendido a realizar la tarea de manera optima (pensamiento divergente).

\section{Método}

\section{Participantes}

Dieciocho participantes, 10 hombres y 8 mujeres, de edades comprendidas entre 21 y 23 años ( $M=22.4$, DT=1.2). Todos los participantes eran sujetos sanos y diestros, y participaron de manera voluntaria. Se les facilitó un formulario de consentimiento de participación en las sesión de grabación EEG.

\section{Procedimiento}

La prueba se realiza durante el año académico 2011/12 y se uso el sistema geodésico de medición aplicado a neuropsicología.

Los participantes tenían que escuchar una locución de 4 minutos de un cuento infantil de naturaleza repetitiva, mientras que visionaban una serie de 10 ilustraciones simultaneas a la locución en las que se les informaba de una serie de gestos que tenía que realizar simultáneamente. Los participantes disponían de 20 minutos tanto para escuchar y memorizar la locución del cuento como para aprender los gestos que la acompañaban simultáneamente.

La tarea experimental consistía en dramatizar el cuento simultáneamente al visionado de las ilustraciones del cuento, a través de la pantalla del ordenador. Cuando el participante cometía un error no se le permitía seguir hasta que la hiciera correctamente.

Se les controlaba el tiempo y el número de errores. Finalmente se recogen los datos en dos momentos; el primer momento durante los primeros 20 minutos (ensayo-error); luego los participantes disponían de 10 minutos para ensayar; después tenían que realizar la tarea lo más rápidamente posible sin cometer errores

\section{Grabación EEG y parámetros de cálculo}

El plano electroencefalográfico consta de 128 canales de registro, con tres puntos de referencia (nariz y región mastoidea derecha e izquierda). Para registrar los movimientos oculares, se efectuó un electro-oculagramma bipolar posicionando dos electrodos respectivamente sobre y debajo y en el interno/externo del canthus ocular para registrar los movimientos oculares horizontales y verticales. La señal EEG fue filtrada entre $0.3 \mathrm{~Hz}$ y $30 \mathrm{~Hz}$, se aplicó un filtro notch a $50 \mathrm{~Hz}$ y manteniendo la impedancia por debajo de $5 \mathrm{~K} \Omega$ por EEG y por debajo de $10 \mathrm{~K} \Omega$ por EOG. Todas las señales fueron mostradas a una frecuencia de $512 \mathrm{~Hz}$. Para obtener una señal EEG "reference free" hemos aplicado el método de referencia promedio polar PARE de 
Junghoefer y col. (1999). Para el calculo de la referencia promedio polar se tomó en consideración que al referir al promedio los datos, la referencia esta sesgada en dirección de la zona mostrada. Por ello, se corrigió este efecto aplicando una interpolación esférica para estimar el voltaje de la superficie no cubierta por el registro electroencefalográfico. La aplicación de este método demuestra una mejora en la resolución espacial de los datos electroencefalográficos sobre todo por la sincronización/desincronización relacionada a eventos (ERS/ERD) del ritmo alpha de $10 \mathrm{~Hz}$. Todos los datos fueron filtrado con una banda de paso de $50 \mathrm{~Hz}$ (fast Fourier low-pass digital filter). Para los análisis de la señal EEG, se utilizó un intervalo de 25 segundos y dentro de éste se evidenció un tiempo de $100 \mathrm{msec}$ antes y $900 \mathrm{msec}$ después de la solución de la tarea. Se calcularon los cambios relacionados a eventos en la banda de frecuencia del ritmo alpha a través de las medias de las sincronizaciones y desincronizaciones relacionadas a eventos; el método propuesto por Pfurtschekker y Aranibar (1977) provee una técnica adecuada para medir el nivel y la distribución topográfica de la actividad oscilatoria cortical durante el rendimiento en las tareas cognitivas (Neuper y Klimesch, 2006). Las investigaciones empíricas demuestran una buena correspondencia entre la topografía ERS/ERD y la actividad cortical subyacente. Por ejemplo, los procesos visuales generan ERD en el área occipital, la palabra en las regiones frontales y temporales, los movimientos de las manos y de los pies se reflejan en actividades ERD en las áreas contralaterales motoras, el funcionamiento de la memoria de trabajo verbal en el área frontal derecha. Se calculó la potencia en las bandas EEG (en microvolt al cuadrado) entre 8 y $12 \mathrm{~Hz}$ por el intervalo de referencia $(R)$ y el intervalo de activación $(A)$ filtrando la señal EEG con el filtro FFT. Sucesivamente se midió la potencia en la banda durante los intervalos de activación sobre todas las pruebas validas; el porcentaje de sincronización/desincronización relacionada a eventos se calculó con la siguiente formula: \%ERS/ERD=[(A-R) x 100]. Se obtuvo el aumento de la frecuencia alpha durante el intervalo de activación a través de la actividad de sincronización (ERS) mientras los valores negativos de la actividad de desincronización (ERD) expresaban la reducción de la frecuencia. Sin embargo, enlazando la banda de potencia de un intervalo de activación con un intervalo pre-estímulo se obtiene, en algunas bandas de frecuencia, una medida de la actividad EEG oscilatoria independiente de las diferencias EEG individuales. Se calculó la fijación de fase (phase locking) entre parejas de electrodos a través del Phase Locking Value, PLV (Lachaux et. al., 1999). El primer algoritmo aplicado filtra los registros EEG y sucesivamente calcula la "convolución" hacia una onda de frecuencia específica. La fase de la convolución nos permite estimar los valores de la PLV sea durante el intervalo de activación, sea durante el intervalo de referencia en cada prueba de baja y alta frecuencia del ritmo alpha. Se concentró el interés en las variaciones de la fijación de fase durante el cambio en la estrategia optima de dramatización del cuento, calculando los valores PLV relacionados a eventos utilizando la misma formula para el calculo del porcentaje de sincronización/desincronización relacionada a eventos.

En consecuencia, los valores positivos PLV muestran un aumento de la actividad durante la fijación de fase, mientras los valores negativos PLV reflejan una reducción de la actividad.

Para indagar las diferencias en ERS/ERD y potenciales relacionados a eventos se realizó un análisis de la varianza (ANOVAs) con medidas repetidas con el factor "tipo de estrategia" como variable entre-sujetos (within-subjects). 


\section{Resultados}

\section{Sincronización/Desincronización relacionada a eventos}

El análisis de la varianza con medidas repetidas por alta y baja frecuencia alpha incluye los siguientes factores entre-sujetos (within-subjects): tipo de estrategia (repetitiva/optimal), hemisferio (diestro/izquierdo) y área de grabación (frontal F3-F4; central C3-C4; parietal P3-P4). Se observa un aumento de la actividad de sincronización y el ANOVA por baja frecuencia alpha, evidenciando una interacción significativa entre el tipo de estrategia y el hemisferio, $F(1,17)=7.21, p<.05$. Como se observa en la figura 1, el uso de la estrategia optima esta caracterizado por ERS mas amplio en el hemisferio diestro, mientras no se detectan ERS amplios durante el uso de estrategias de tipo repetitivo. En el hemisferio izquierdo el ANOVA no muestra diferencias significativas. Respecto a la banda de frecuencia alpha mas ancha, se detecta un solo efecto principal respecto al factor "hemisferio", $F(1,17)=8.14, p<.05$. También se puede observar un porcentaje mayor de ERS anchos (75\%) en el hemisferio diestro respecto al diestro (25\%). No se relevan otros efectos principales.

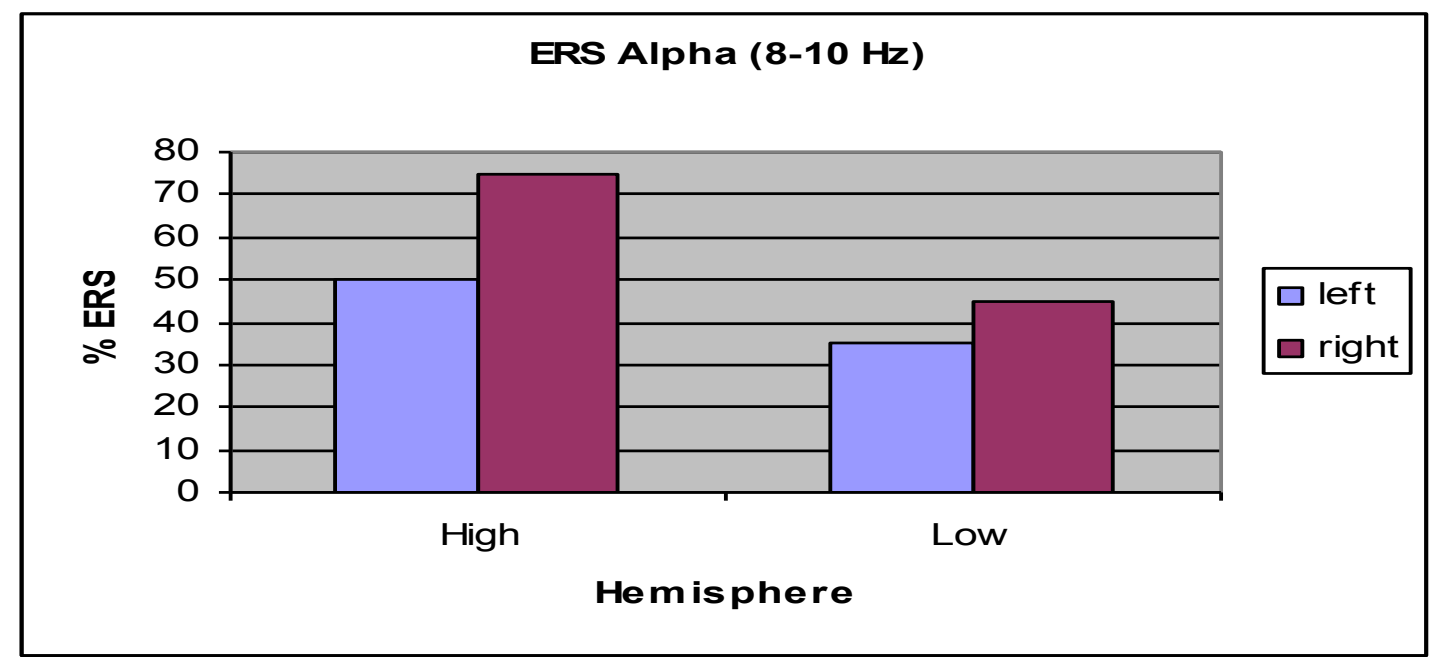

Figura1. Actividad ERS de tipo Alpha en los dos hemisferios

Se realiza el análisis de los valores de la fijación de fase (PLV) relacionados a eventos en tres pasos diferentes. Primero se analiza la fijación de fase inter-hemisférica en las áreas frontales, centrales y parietales; se realiza el análisis de la varianza con medidas repetidas por el factor "tipo de estrategia" sobre diferentes parejas de electrodos (frontal F3, F4; central C3, C4; parietal P3, P4) y para los dos intervalos del ritmo alpha (8-10 Hz, 10-12 Hz); sucesivamente se analiza la fijación de fase frontal-parietal en la distancia intra-hemisférica (izquierda F3-P3; diestra F4-P4), calculando el análisis de la varianza con medidas repetidas por el factor hemisferio y tipo de estrategia. De la misma manera se calcula la fijación de fase intra-hemisférica entre las áreas anteriores y posteriores (hemisferio izquierdo: PF3-F3, FC5-F3, FC1-F3, PF3-FC1; hemisferio diestro: PF4-F4, FC6-F4, FC2-F4, PF4-FC6, PF4-FC2). En el ritmo alpha de baja frecuencia muestra una interacción significativa entre tipo de estrategia utilizada y hemisferio: $F(1,17)=6.76, p<.05$. Los valores mas altos de la PVL se refieren al hemisferio diestro durante la ejecución de la tarea con estrategia optimal (respecto al uso de la estrategia de naturaleza repetitiva), pero no se evidencia ninguna diferencia significativa entre los valores de la PVL relacionados a eventos en el hemisferio izquierdo. En la figura 2 se puede observar que las diferencias subyacentes los valores de la PVL en el hemisferio diestro se deben a las actividades grabadas en PF4-FC6 y PF4-FC2. En las frecuencias alpha mas amplias $(10-12 \mathrm{~Hz})$ 
hemos encontrado un efecto principal "hemisferio" $F(1,17)=6.3, p<.05$, con lo cual podemos presumir que hay una mayor actividad de fijación de fase (phase locking) en el hemisferio diestro respecto al izquierdo, tal como se muestra en la figura 3.

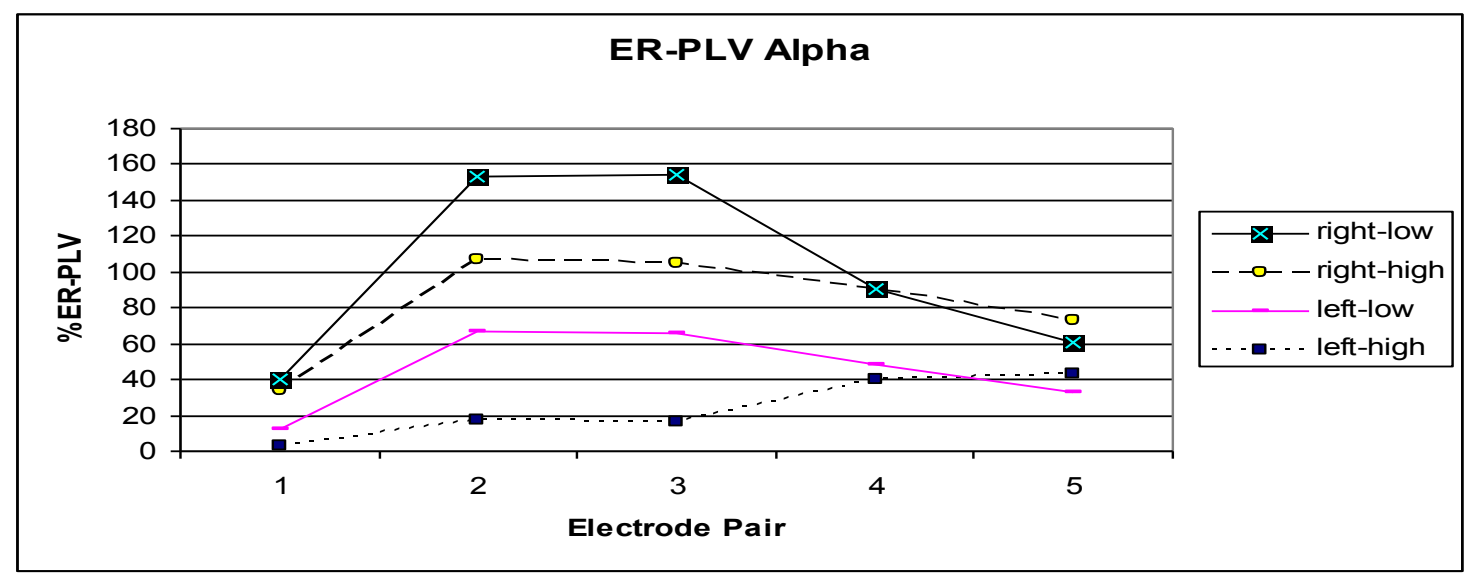

Figura 2. Interacción entre hemisferio, estrategia utilizada y valores PVL relacionados a eventos por el ritmo Alpha con baja frecuencia

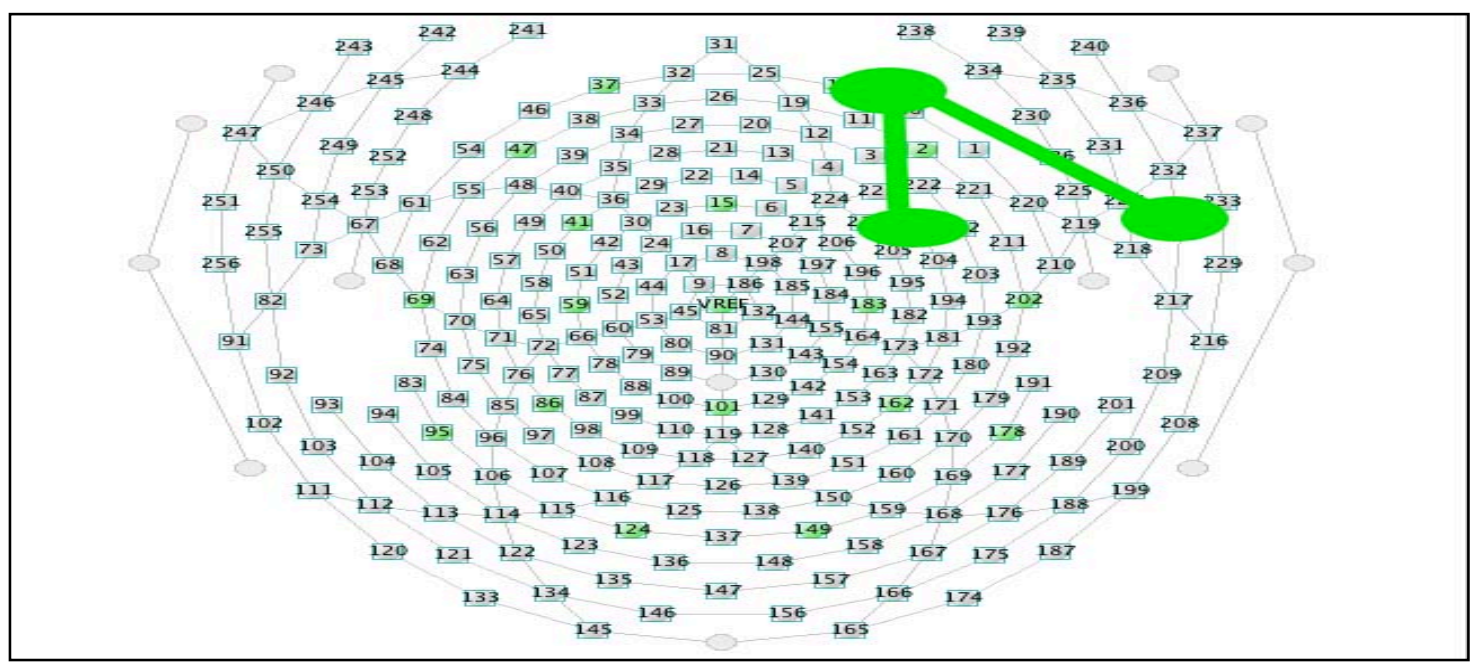

Figura 3. Mapa topográfica de las grabaciones EEG con indicación de la fijación de fase en las áreas prefrontal y pre-central

\section{Discusión}

Los resultados confirman la hipótesis relativa a la presencia de patrones neurofisiológicos diferentes que subyacen la diferente capacidad, creativa o no, de solucionar un problema también cuando la naturaleza de éste resulta ser de tipo repetitivo. Durante el pensamiento creativo y divergente hemos evidenciado una alta fase de sincronización por el hemisferio derecho. A la luz de los resultados, podemos decir que el pensamiento creativo puede estar asociado con diferentes patrones de actividad EEG de tipo oscilatorio, el ritmo alpha. Eso vale para el ritmo alpha de 8-10 
$\mathrm{Hz}$ que, como sabemos de la literatura, esta asociado a la actividad de atención noespecifica y en condición de alerta generalizada (Klimesch, 1999); sin embargo el ritmo alpha con frecuencia de $10-12 \mathrm{~Hz}$ esta asociado a las actividades especificas (memoria semántica, pruebas de inteligencia) que producen actividad sincronizada y fijación de fase (phase locking) en el hemisferio diestro (Grabner et al., 2004; Neubauer, Grabner, \& Fink, 2005). Como también se evidencia en anteriores trabajos sobre la originalidad y el pensamiento creativo (Grabner, Fink, \& Neubauer, 2007) hemos detectado como el uso de estrategias optimas y de naturaleza no repetitiva produzcan una relevante actividad de sincronización relacionada a eventos en el hemisferio diestro, así como hemos evidenciado un aumento de la actividad alpha en el mismo hemisferio. Estos datos confirman los datos existente en la literatura sobre la capacidad del hemisferio diestro de trabajar en modalidad paralela y holística, respecto a la modalidad lógica y analítica del hemisferio izquierdo. Además, como describen los autores (Kris, 1952; Mednick, 1962; Mendelsohn, 1976) es probable que el hemisferio diestro sea capaz de trabajar según un proceso cognitivo primario libre de asociaciones. Otra evidencia experimental es aquella relativa a los valores de la fijación de fase (PVL) intra-hemisférica relacionada a eventos: el uso de estrategias optimas en la resolución de la tarea revela valores altos de PVL en la breve distancia entre las ares frontal-polar (FP) y pre-central (FC). Estos resultados confirman la hipótesis según la cual la corteza frontal representa una región cerebral determinante por el pensamiento creativo: las áreas de la corteza frontal subyacen la flexibilidad del pensamiento creativo en la solución de problemas, proveen los recursos atencionales adecuados sea en la evaluación de una nueva idea/solución, sea para generar nuevos contenidos semánticos (Howard-Jones et al., 2005). En conclusión podemos decir que nuestros resultados se alinean con las evidencias experimentales de otras investigaciones existentes en la literatura científica y en particular podemos confirmar que los procesos creativos generan precisos patrones de actividad EEG oscilatoria: en lo especifico, el pasaje de una estrategia de tipo "repetitivo" a una que nosotros definimos como optima, esta caracterizado por un aumento del ritmo alpha y un aumento de la actividad sincronizada del hemisferio diestro. Con esta ultima consideración subrayamos la importancia del hemisferio diestro y de las redes corticales involucradas en el proceso creativo en las capacidades cognitivas de solución de problemas.

\section{Referencias}

Bowden, E.M., \& Jung-Beeman, M. (1998). Getting the right idea: Semantic activation in the right hemisphere may help solve insight problems. Psychological Science, 9, 435-440.

Bowden, E.M., \& Jung-Beeman, M. (2003). Aha! Insight experience correlates with solution activation in the right hemisphere. Psychonomic Bulletin \& Review, 10, 730-737.

Bowden, E.M., Jung-Beeman, M., Fleck, J., \& Kounios, J. (2005). New approaches to demystifying insight. Trends in Cognitive Sciences, 9, 322-328.

Dietrich, A. (2004). The cognitive neuroscience of creativity. Psychonomic Bulletin \& Review, 11, 1011-1026.

Fiorini, M. (2012). Creatividad entre biología y cultura. Andaluciaeduca, 75, 102-105. 
Fiorini, M. (2012). Didáctica Metacognitiva y Empowerment Social. Andaluciaeduca, 84, 7-7.

Fiorini, M., \& García-Ramírez, J.M. (2013). Cap. 5: Técnicas de grupo y creatividad aplicadas en el ámbito universitario, en Villena, M.D., Muñoz, A. Recursos para tutoría en el aula universitaria. Granada: Editorial Universidad de Granada.

Grabner, R.H., Fink, A., Stipacek, A., Neuper, C., \& Neubauer, A.C. (2004). Intelligence and working memory systems: Evidence of neural efficiency in alpha band ERD. Cognitive Brain Research, 20, 212-225.

Guilford, J.P. (1950). Creativity. American Psychologist, 5, 444-454.

Guilford, J.P. (1967). The nature of human intelligence. New York:McGraw-Hill.

Howard-Jones, P.A., Blakemore, S.J., Samuel, E.A., Summers, I.R., \& Claxton, G. (2005). Semantic divergence and creative story generation: An fMRI investigation. Cognitive Brain Research, 25, 240-250.

Jausovec, N. (2000). Differences in cognitive processes between gifted, intelligent, creative, and average individuals while solving complex problems: An EEG study. Intelligence, 28, 213-237.

Junghoefer, M., Elbert, T., Tucker, D.M., Braun, C., (1999). "The polar average referenced effect: a bias in estimatine the head surface integral in eeg recording. Electroencephalography an clinical Neurophysiology, 98, 422-432.

Klimesch, W., Doppelmayr, M., Schwaiger, J., Auinger, P., \& Winkler, T. (1999). "Paradoxical" alpha synchronization in a memory task. Cognitive Brain Research, 7, 493-501.

Kris, E. (1952). Psychoanalytic Explorations in Art. New York: International Universities Press.

Lachaux, J.P., Rodriguez, E., Martinerie, J., \& Varela, F.J. (1999). Measuring phase synchrony in brain signals. Human Brain Mapping, 8, 194-208.

Martindale, C. (1999). Biological bases of creativity. In R. Sternberg (Ed.), Handbook of creativity (137-152). Cambridge: Cambridge University Press.

Martindale, C., \& Hasenfus, N. (1978). EEG differences as a function of creativity, stage of the creative process, and effort to be original. Biological Psychology, 6 , 157-167.

Martindale, C., Hines, D., Mitchell, L., \& Covello, E. (1984). EEG alpha asymmetry and creativity. Personality and Individual Differences, 5, 77-86.

Mednick, S.A. (1962). The associative basis of the creative process. Psychological Review, 69, 220-232.

Mendelsohn, G.A. (1976). Associative and attentional processes in creative performance. Journal of Personality, 44, 341-369. 
Molle, M., Marshall, L., Wolf, B., Fehm, H.L., \& Born, J. (1999). EEG complexity and performance measures of creative thinking. Psychophysiology, 36, 95-104.

Neuper, C., \& Klimesch, W. (Eds.) (2006). Progress in Brain Research: Vol. 159. Event-related dynamics of brain oscillations. Amsterdam: Elsevier.

Neubauer, A.C., Grabner, R.H., Fink, A., \& Neuper, C. (2005). Intelligence and neural efficiency: Further evidence of the influence of task content and sex on the brain-IQ relationship. Cognitive Brain Research, 25, 217-225.

Pfurtscheller, G., \& Aranibar, A. (1977). Event-related cortical desynchronization detected by power measurements of scalp EEG. Electroencephalography and Clinical Neurophysiology, 42, 817-826. 